Research Paper

\title{
Changes in Disease Failure Risk of Nasopharyngeal Carcinoma over Time: Analysis of 749 Patients with Long-Term Follow-Up
}

\author{
Xu Liu1 ${ }^{*}$, Ling-Long Tang1 ${ }^{*}$, Xiao-Jing Du1 ${ }^{*}$, Wen-Fei Li1 ${ }^{1}$, Lei Chen ${ }^{1}$, Guan-Qun Zhou1 ${ }^{1}$, Rui Guo ${ }^{1}$, Qing Liu ${ }^{2}$, \\ Ying Sun ${ }^{1}$, Jun Ma ${ }^{1 凶}$ \\ 1. Department of Radiation Oncology, Sun Yat-sen University Cancer Center, State Key Laboratory of Oncology in South China, Collaborative Innovation \\ Center for Cancer Medicine, Canton 510060, China. \\ 2. Department of Medical Statistics and Epidemiology, School of Public Health, Sun Yat-sen University, Canton 510060, China. \\ * Xu Liu, Ling-Long Tang and Xiao-Jing Du contributed equally to this work. \\ $\triangle$ Corresponding author: Jun Ma, Professor, Department of Radiation Oncology, Sun Yat-sen University Cancer Center, No. 651 Dongfeng Road East, Canton \\ 510060, Guangdong, China. Telephone: +86-20-87343469 Fax: +86-20-87343295 E-mail: majun2@mail.sysu.edu.cn. \\ (C) Ivyspring International Publisher. This is an open access article distributed under the terms of the Creative Commons Attribution (CC BY-NC) license \\ (https:// creativecommons.org/licenses/by-nc/4.0/). See http://ivyspring.com/terms for full terms and conditions.
}

Received: 2016.08.04; Accepted: 2016.10.17; Published: 2017.02.10

\begin{abstract}
Background: The changes in the risk of disease failure over time in nasopharyngeal carcinoma (NPC) remains unknown.

Methods: We analyzed 749 patients treated with intensity-modulated radiotherapy in a single center. The annual hazard rates of disease failure (recurrence or death from any cause) were estimated using the life-table method.

Results: In total, 41 (5.5\%), $22(2.9 \%)$ and 129 (17.2\%) patients developed local, regional and distant recurrences, respectively; $149(19.9 \%)$ patients died. Of the 600 patients who were alive at the last follow-up, 496 (82.7\%) had follow-up times longer than 6 years. The 6-year failure-free survival rate was $74.8 \%$. Older age ( $>50$ years) and advanced stage (III-IVB) were independent risk factors for disease failure in multivariate analysis. The hazard curve for failure risk in the whole cohort showed a sharp peak at 2 years, changed to a gradually decreasing plateau between years 3 and 5 and then declined sharply. Subgroup analyses revealed similar hazard curves in both sexes. However, the patterns of hazard curve significantly differed between high-risk $(>50$ years or stage III-IVB) and low-risk ( $\leq 50$ years or stage $\mathrm{I}-\mathrm{II}$ ) patients.

Interpretation: The failure hazard rate in NPC didn't decline in a linear manner, but displayed a sharp peak at 2 years. The patterns of hazard function significantly differed between patients with different age and stage. Further studies are warranted to confirm our results.
\end{abstract}

Key words: Nasopharyngeal neoplasms; Prognosis; Recurrence; Mortality; Hazard function.

\section{Introduction}

In the field of oncology, the predominant way of reporting outcome is survival curves. A survival curve describes the cumulative event-free time distribution at a given time point after diagnosis or accrual in clinical trials. However, it does not give direct information regarding changes in event probability over time. Such information is highlighted using the hazard function, which provides the event incidence rate at any instant among survivors up to that point in time. It is also known as the conditional failure rate or time-specific mortality rate. The time distribution of failure risk has been best studied in breast cancer, and this has provided meaningful insights into tumor biology and shed light on the clinical management of breast cancer.[1-5]

Nasopharyngeal carcinoma (NPC) is prevalent 
in southern China, Southeast Asia, North Africa, the Middle East and Alaska.[6, 7] Radiotherapy with or without chemotherapy is the primary treatment for NPC. The long-term failure-free survival (FFS) rate in NPC has been estimated to be $67 \%-79 \%$.[8, 9] To the best of our knowledge, no study has investigated the change in failure risk over time in NPC. Thus, the aim of this study was to analyze the hazard function of disease failure in a large cohort of NPC patients who underwent long-term follow-up.

\section{Patients and Methods}

\section{Patient characteristics}

We retrospectively reviewed the records of all 749 newly diagnosed, biopsy-proven, non-metastatic (stage I-IVB) NPC patients who were treated with intensity-modulated radiotherapy (IMRT) at our center between January 2003 and December 2007. The clinical features of these patients are shown in Table 1.

Table 1. Clinical features of the 749 nasopharyngeal carcinoma patients in the study.

\begin{tabular}{|c|c|}
\hline Characteristics & No. of patients (\%) \\
\hline \multicolumn{2}{|l|}{ Age (years) } \\
\hline$\leq 50$ & $553(73.8)$ \\
\hline$>50$ & $196(26.2)$ \\
\hline \multicolumn{2}{|l|}{ Sex } \\
\hline Male & $580(77.4)$ \\
\hline Female & 169 (22.6) \\
\hline \multicolumn{2}{|l|}{ Pathology type } \\
\hline Keratinizing squamous cell carcinoma & $5(0.7)$ \\
\hline Nonkeratinizing carcinoma & $744(99.3)$ \\
\hline \multicolumn{2}{|l|}{ Chemotherapy } \\
\hline Yes & $535(71.4)$ \\
\hline No & $214(28.6)$ \\
\hline \multicolumn{2}{|l|}{ T category ${ }^{\dagger}$} \\
\hline T1 & $177(23.6)$ \\
\hline$T 2$ & $140(18.7)$ \\
\hline T3 & $264(35.2)$ \\
\hline T4 & $168(22.4)$ \\
\hline \multicolumn{2}{|l|}{$\mathrm{N}$ category ${ }^{\dagger}$} \\
\hline No & $184(24.6)$ \\
\hline N1 & 409 (54.6) \\
\hline N2 & $106(14.2)$ \\
\hline N3 & $50(6.7)$ \\
\hline \multicolumn{2}{|l|}{ Stage $^{\dagger}$} \\
\hline$I$ & $78(10.4)$ \\
\hline II & $179(23.9)$ \\
\hline III & $282(37.7)$ \\
\hline$I V A-I V B$ & $210(28.0)$ \\
\hline
\end{tabular}

$\mathrm{T}$, tumor; $\mathrm{N}$, node.

†According to the $7^{\text {th }}$ Union for International Cancer Control/American Joint Committee on Cancer staging system.

All patients completed a pretreatment evaluation including complete patient history, physical examination, hematology and biochemistry profiles, nasopharynx and neck magnetic resonance imaging (MRI), chest radiography, abdominal ultrasonography and single photon emission computed tomography whole-body bone scan. Positron-emission tomography/computed tomography (PET/CT) was performed in 162 of the 749 patients $(21.6 \%)$. All patients were restaged according to the 7 th Union for International Cancer Control/American Joint Committee on Cancer (UICC/AJCC) staging system.[10] The study was performed after the ethical approval from Institutional Review Board of Sun Yat-sen University Cancer Center. Informed consent was obtained from each patient for their consent to have their information used in research without affecting their treatment option or violating their privacy.

\section{Treatment}

All patients underwent radical radiation therapy. The nasopharyngeal and upper neck target volumes were treated using IMRT for the entire treatment course. The lower neck was treated with a conventional anterior or anteroposterior opposing cervical technique. Further details of the IMRT techniques used at our center have been reported previously.[11]

During the study period, our institutional guidelines recommended no chemotherapy for stage I to IIA NPC, concurrent chemoradiation therapy for stage IIB and concurrent chemoradiation therapy with or without neoadjuvant/adjuvant chemotherapy for stage III to IVA-B NPC, as defined by the 6th edition of the UICC/AJCC staging system. In total, $86.2 \%$ of stage III-IVB patients (424 of 492) received chemotherapy. When possible, salvage treatments such as intracavitary brachytherapy, surgery and chemotherapy were provided in the event of documented recurrence or persistent disease.

\section{Follow-up}

Patients attended follow-up visits every 2-3 months during the first 2 years; every 3-6 months during years 3-5 and annually thereafter or until death. Fiberoptic endoscopy, CT or MRI scans of the head and neck were performed every 3 months during the first year and annually during years 2-5. All local relapses were diagnosed by fiberoptic endoscopy and biopsy or CT/MRI of the nasopharynx and skull base. Regional recurrences were diagnosed by fine needle aspiration, CT/MRI or pathological examination after surgery. Distant metastases were diagnosed on the basis of clinical symptoms and the findings of physical examinations and imaging studies, including chest X-ray, bone scan, MRI, CT and abdominal sonography. Additional tests were ordered whenever necessary. 


\section{Statistical analysis}

FFS was considered as the time elapsed from the first day of treatment to the date of disease failure, defined as locoregional or distant recurrence or death from any cause, whichever occurred first; the time was censored at the date of the last follow-up evaluation. Actuarial rates were estimated by the Kaplan-Meier method, and survival curves were compared using the log-rank test.[12] Multivariate analyses with the Cox proportional hazards model were used to test for independent significance by backward elimination of insignificant explanatory variables.[13] The failure risk over time was studied by using the life-table method to estimate the annual failure hazard rate, i.e., the conditional probability of manifesting disease failure in a year given that the patient is clinically free of any failure at the beginning of the year.

The criterion for statistical significance was set at $a<0.05$. All $P$ values were based on two-sided tests. We used Statistical Product and Service Solutions (SPSS) version 20.0 (IBM Corporation, Armonk, NY, USA) to perform modeling and statistical calculations.

\section{Results}

\section{Follow-up and patterns of disease failure}

The median duration of follow-up for the entire cohort was 81.4 months (range, 3.1-126.5 months). A total of $41(5.5 \%), 22(2.9 \%)$ and $129(17.2 \%)$ local, regional and distant recurrence events were detected in the entire cohort during follow-up, respectively; $149(19.9 \%)$ patients died. For the 600 patients alive at the last follow-up, the median follow-up time was 87.1 months (range, 5.8-126.5 months). In 593 (98.8\%), $496(82.7 \%)$ and $334(55.7 \%)$ of these patients, the follow-up time was longer than 5, 6 and 7 years, respectively. Thus, we restricted the hazard function analysis to 6 years of follow-up in order to minimize possible bias due to inadequate follow-up. Among all 197 patients developed disease failure, 188 (95.4\%) experienced the first disease failure during the first 6 years of follow-up. The patterns of disease failure over time during 6 years of follow-up are shown in Table 2. The 6-year FFS rate was $74.8 \%$.

\section{Survival analysis}

On univariate analysis, the 6-year FFS was found to be significantly poorer in patients older than 50 years than in patients younger than 50 years $(65.7 \%$ vs. $78.0 \%, P<0.001$ ). Inferior 6-year FFS was also observed in patients with advanced stage disease (stage III-IVB vs. stage I-II, $66.0 \%$ vs. $91.7 \%, P<$ 0.001). In contrast, sex and pathological type were not significant prognostic factors $(P=0.401$ and 0.938 , respectively). Multivariate analysis incorporating host factors (i.e., age, sex), tumor factors (i.e., stage) and therapeutic intervention (i.e., radiotherapy alone or chemoradiotherapy) confirmed that age and stage were independent prognostic factors for FFS $(P<$ 0.001 for both).

\section{Hazard function}

The hazard curve for disease failure in the whole cohort peaked at 2 years, changed to a slowly decreasing plateau between 3 and 5 years and then declined sharply (Figure 1). Then we conducted subgroup analyses according to various clinical features. The hazard curve was similar in both sexes, but exhibited more pronounced variations in female patients (Figure 2A). Interestingly, the hazard curve displayed different patterns in patients with different ages (Figure 2B). In patients older than 50 years, the hazard curve had two peaks. The first peak occurred at 2 years, and the second peak between 4 and 5 years. In patients under the age of 50, the hazard rate was much lower and lacked a sharp peak. Analysis of the graphs in early (stage I-II) and locoregionally advanced (stage III-IVB) disease showed that the hazard function in the whole cohort was dominated by that in patients with locoregionally advanced disease (Figure 2C). The hazard rate in patients with early disease was less than $2.5 \%$ during the 6 years of follow-up.

Table 2. Patterns of disease failure over time in patients with nasopharyngeal carcinoma.

\begin{tabular}{|c|c|c|c|c|c|c|}
\hline Years at follow-up & No. at risk & $\begin{array}{l}\text { No. of patients developed the } 1^{\text {st }} \\
\text { disease failure* }\end{array}$ & Percentage $(\%)$ & $\begin{array}{l}\text { No. of locoregional } \\
\text { recurrences }\end{array}$ & $\begin{array}{l}\text { No. of distant } \\
\text { recurrences }\end{array}$ & No. of death \\
\hline 1 & 749 & 50 & 6.7 & 9 & 38 & 12 \\
\hline 2 & 698 & 53 & 7.6 & 18 & 37 & 29 \\
\hline 3 & 645 & 29 & 4.5 & 11 & 18 & 29 \\
\hline 4 & 613 & 26 & 4.2 & 6 & 21 & 31 \\
\hline 5 & 587 & 22 & 3.8 & 6 & 10 & 19 \\
\hline 6 & 563 & 8 & 1.5 & 2 & 3 & 15 \\
\hline Total & - & 188 & - & 52 & 127 & 135 \\
\hline
\end{tabular}

No., number.

* Disease failure was defined as locoregional or distant recurrence or death from any cause. 


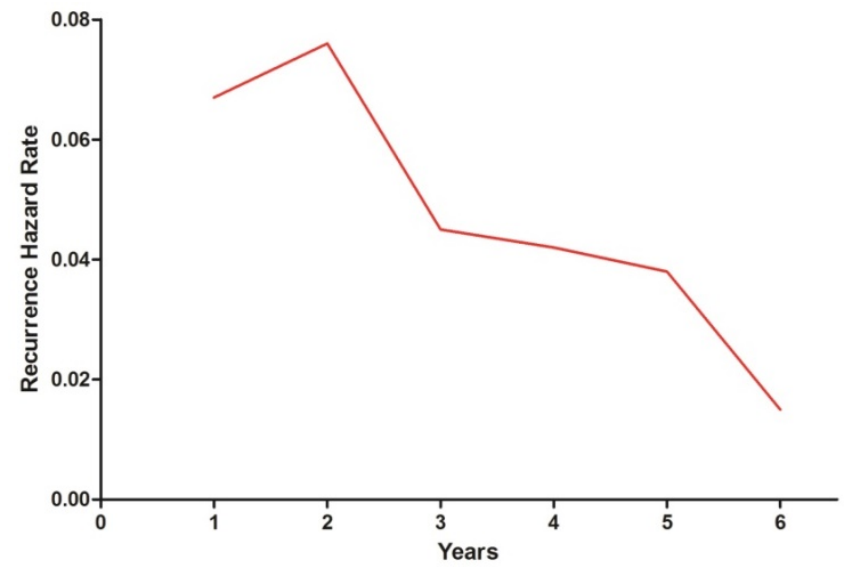

Figure 1. Annual hazard rates for disease failure in nasopharyngeal carcinoma.

A

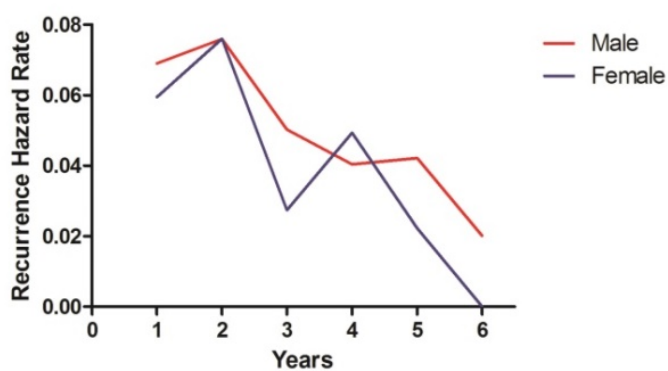

B

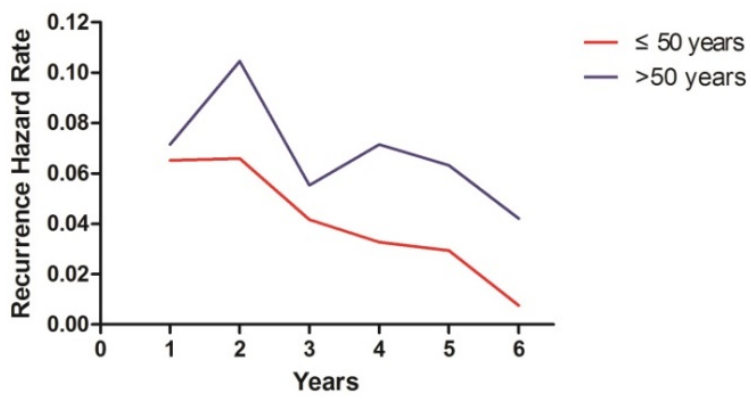

C

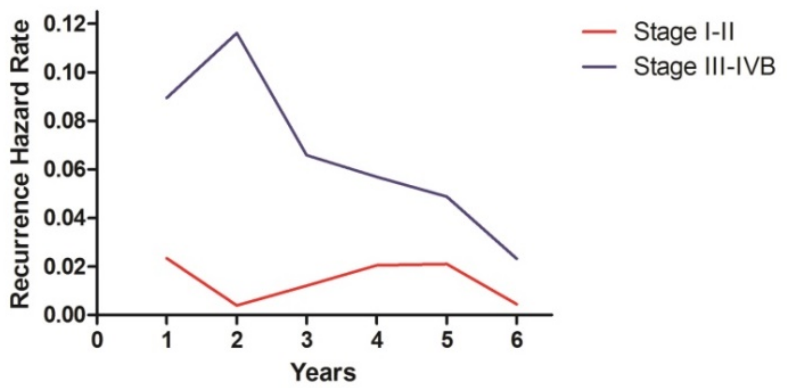

Figure 2. Annual hazard rates for disease failure in nasopharyngeal carcinoma stratified by sex (A), age (B) and stage (C).

\section{Discussion}

In this study, we analyzed the change in failure risk over time in NPC patients and found a counterintuitive pattern of hazard function. The NPC failure hazard rate did not decline in a linear manner, but showed a sharp peak at 2 years. This phenomenon challenges the explanation obtained by classical Gompertzian or exponential kinetics of tumor growth, but may be explained by the tumor dormancy hypothesis. According to this hypothesis, microscopic tumor foci may be in different biological steady states and do not lead to tumor growth (e.g. a balance of cell death and division) in the absence of growth factors or in the presence of anti-angiogenic factors.[14-16]

Research has shown that surgery to remove tumors may also remove sources of angiogenesis inhibitors or lead to the appearance of growth factors in circulation in response to surgical wounding, which may terminate the dormancy of the tumor foci and result in accelerated recurrences.[17, 18] Our results suggested that the tumor dormancy-reactivation hypothesis might be applicable to NPC. $[19,20]$ However, this hypothesis needs to be tested in future studies.

Multivariate analysis confirmed that older age ( $>50$ years) and advanced stage (stage III-IVB) were independent risk factors for disease failure in NPC. The hazard function stratified by age and stage displayed significantly different patterns. Similarly, distinct hazard functions have been well demonstrated in breast cancer for large versus small tumors, positive versus negative lymph nodes, high versus low tumor grades,[21] intrinsic molecular breast cancer subtypes, MammaPrint prognostic molecular signatures and Oncotype DX prognostic scores.[4] Moreover, the hazard function stratified by different phenotypes and genotypes of breast cancer had good concordance.[4] In general, hazard curves for recurrences among high-risk tumors (e.g., nonluminal A, Mammaprint poor signature and Oncotype high-risk score) show a sharp peak soon after the initial diagnosis, similar to estrogen receptor (ER)-negative cancers.[4] Conversely, hazard curves for low-risk tumors (e.g., luminal A, Mammaprint good signature and Oncotype low- and intermediate-risk scores) lack a sharp peak, similar to ER-positive tumors.[4] Thus, it is reasonable to speculate that there might be intrinsic biological differences between high-risk $(>50$ years or stage III-IVB) and low-risk ( $\leq 50$ years or stage I-II) NPCs, and further studies are warranted.

Besides the theoretical value, our results are of clinical importance at two levels. First, the understanding of the time distribution of failure risk could help oncologists to design more appropriate follow-up strategies. For example, we showed that the failure risk in patients with early disease (stage I-II) was lower than $2.5 \%$ during the first 6 years of 
follow-up. This raised the question of whether it is reasonable to use the same intensive follow-up strategy in patients with early and locoregionally advanced disease. Second, the hazard rate of NPC varies over time, suggesting that the biological mechanisms responsible for early and late disease failures differ and may therefore respond differently to the same treatment. For example, Berry et al. have analyzed the data of 6,644 patients from three randomized trials coordinated by Cancer and Leukemia Group B, and revealed that high-dose versus low-dose adjuvant chemotherapy reduced the risk of recurrence or death by $55 \%$ in the first year and $30 \%$ in the second year, with no advantage after 3 years.[22] Researchers have proposed that we should take into account the time-dependent effects of adjuvant therapy in breast cancer.[4] Similarly, the effects of therapy might also vary over time in NPC, and this possibility needs to be confirmed in future studies.

In this study, we only analyzed the hazard function during 6 years of follow-up, since $82.7 \%$ of live patients had been followed up for longer than 6 years. Although we can calculate the hazard rate beyond 6 years, we consider that possible bias would influence the interpretation of the results due to inadequate follow-up. We intend to update our results in the future after ten years of follow-up.

In conclusion, the hazard rate of disease failure did not decline in a linear manner over time, but displayed a sharp peak at 2 years, changed to a gradually decreasing plateau between years 3 and 5 and then declined sharply. Older age ( $>50$ years) and advanced stage (stage III-IVB) were independent risk factors for disease failure in NPC. The patterns of hazard function significantly differed between patients with different age and stage. Understanding the underlying mechanism of the time distribution of failure risk would help develop more effective treatment and follow-up strategies for NPC.

\section{Abbreviations}

NPC: nasopharyngeal radiotherapy; MRI: magnetic resonance imaging; PET/CT: positron-emission tomography/computed tomography; UICC/AJCC: Union for International Cancer Control/American Joint Committee on Cancer; SPSS: Statistical Product and Service Solutions; ER: estrogen receptor.

\section{Acknowledgements}

This work was supported by grants from the National Science \& Technology Pillar Program during the Twelfth Five-year Plan Period (2014BAI09B10), the
Science and Technology Project of Guangzhou City, China (No. 14570006), the Planned Science and Technology Project of Guangdong Province (No. 2013B020400004) and the National Natural Science Foundation of China $(81572658,81572962)$.

\section{Competing Interests}

The authors have declared that no competing interest exists.

\section{References}

1. Saphner T, Tormey DC, Gray R. Annual hazard rates of recurrence for breast cancer after primary therapy. J Clin Oncol. 1996; 14: 2738-46.

2. Demicheli R, Miceli R, Moliterni A, Zambetti M, Hrushesky WJ, Retsky MW, et al. Breast cancer recurrence dynamics following adjuvant CMF is consistent with tumor dormancy and mastectomy-driven acceleration of the metastatic process. Ann Oncol. 2005; 16: 1449-57.

3. Dillekas H, Transeth M, Pilskog M, Assmus J, Straume O. Differences in metastatic patterns in relation to time between primary surgery and first relapse from breast cancer suggest synchronized growth of dormant micrometastases. Breast Cancer Res Treat. 2014; 146: 627-36.

4. Jatoi I, Anderson WF, Jeong JH, Redmond CK. Breast cancer adjuvant therapy: time to consider its time-dependent effects. J Clin Oncol. 2011; 29: 2301-4.

5. Dignam JJ. Some additional thoughts on time-varying treatment effects in breast cancer. J Clin Oncol. 2011; 29: 4469-70; author reply 70.

6. Chua ML, Wee JT, Hui EP, Chan AT. Nasopharyngeal carcinoma. Lancet. 2016; 387: 1012-24.

7. Wei KR, Zheng RS, Zhang SW, Liang ZH, Ou ZX, Chen WQ. Nasopharyngeal carcinoma incidence and mortality in China in 2010. Chin J Cancer. 2014; 33: 381-7.

8. Wolden SL, Chen WC, Pfister DG, Kraus DH, Berry SL, Zelefsky MJ. Intensity-modulated radiation therapy (IMRT) for nasopharynx cancer: update of the Memorial Sloan-Kettering experience. Int J Radiat Oncol Biol Phys. 2006; 64: 57-62.

9. Tham IW, Hee SW, Yeo RM, Salleh PB, Lee J, Tan TW, et al. Treatment of nasopharyngeal carcinoma using intensity-modulated radiotherapy-the national cancer centre singapore experience. Int J Radiat Oncol Biol Phys. 2009; 75: 1481-6.

10. Edge SB, Byrd DR, Compton CC, Fritz AG, Greene FL, Trotti A. American Joint Committee on Cancer cancer staging manual. 7th ed. New York, NY: Springer; 2010.

11. Lai SZ, Li WF, Chen L, Luo W, Chen YY, Liu LZ, et al. How does intensity-modulated radiotherapy versus conventional two-dimensional radiotherapy influence the treatment results in nasopharyngeal carcinoma patients? Int J Radiat Oncol Biol Phys. 2011; 80: 661-8.

12. Kaplan EL, Meier P. Nonparametric Estimation from Incomplete Observations. Journal of the American Statistical Association. 1958; 53: 457-81.

13. Cox DR. Regression Models and Life-Tables. Journal of the Royal Statistical Society Series B (Methodological). 1972; 34: 187-220.

14. Giancotti FG. Mechanisms Governing Metastatic Dormancy and Reactivation. Cell. 2013; 155: 750-64.

15. Gunduz N, Fisher B, Saffer EA. Effect of surgical removal on the growth and kinetics of residual tumor. Cancer Res. 1979; 39: 3861-5.

16. Holmgren L, O'Reilly MS, Folkman J. Dormancy of micrometastases: balanced proliferation and apoptosis in the presence of angiogenesis suppression. Nat Med. 1995; 1: 149-53.

17. Tagliabue E, Agresti R, Carcangiu ML, Ghirelli C, Morelli D, Campiglio M, et al. Role of HER2 in wound-induced breast carcinoma proliferation. Lancet. 2003; 362: 527-33.

18. Retsky M, Demicheli R, Hrushesky WJ. Does surgery induce angiogenesis in breast cancer? Indirect evidence from relapse pattern and mammography paradox. Int J Surg. 2005; 3: 179-87.

19. Naumov GN, Townson JL, MacDonald IC, Wilson SM, Bramwell VH, Groom $\mathrm{AC}$, et al. Ineffectiveness of doxorubicin treatment on solitary dormant mammary carcinoma cells or late-developing metastases. Breast Cancer Res Treat. 2003; 82: 199-206.

20. Braun S, Kentenich C, Janni W, Hepp F, de Waal J, Willgeroth F, et al. Lack of effect of adjuvant chemotherapy on the elimination of single dormant tumor cells in bone marrow of high-risk breast cancer patients. J Clin Oncol. 2000; 18: 80-6.

21. Anderson WF, Jatoi I, Devesa SS. Distinct breast cancer incidence and prognostic patterns in the NCI's SEER program: suggesting a possible link between etiology and outcome. Breast Cancer Res Treat. 2005; 90: 127-37.

22. Berry DA, Cirrincione C, Henderson IC, Citron ML, Budman DR, Goldstein LJ, et al. Estrogen-receptor status and outcomes of modern chemotherapy for patients with node-positive breast cancer. JAMA. 2006; 295: 1658-67. 